\title{
Energy Efficient Cell Management by Flow Scheduling in Ultra Dense Networks
}

\author{
Guolin Sun, Prince Clement Addo, Guohui Wang, Guisong Liu \\ School of Computer Science and Engineering, \\ University of Electronic Science and Technology of China \\ Chengdu, 611731 - CHINA \\ [e-mail: guolin.sun@uestc.edu.cn] \\ *Corresponding author: Guolin Sun
}

Received January 18, 2016; revised June 23, 2016; accepted August 3, 2016;

published September 30, 2016

\begin{abstract}
To address challenges of an unprecedented growth in mobile data traffic, the ultra-dense network deployment is a cost efficient solution to off-load the traffic over other small cells. However, the real traffic is often much lower than the peak-hour traffic and certain small cells are superfluous, which will not only introduce extra energy consumption, but also impose extra interference onto the radio environment. In this paper, an elastic energy efficient cell management scheme is proposed based on flow scheduling among multi-layer ultra-dense cells by a SDN controller. A significant power saving was achieved by a cell-level energy manager. The scheme is elastic for energy saving, adaptive to the dynamic traffic distribution in the office or campus environment. In the end, the performance is evaluated and demonstrated. The results show substantial improvements over the conventional method in terms of the number of active BSs, the handover times, and the switches of BSs.
\end{abstract}

Keywords: Software defined networks, ultra-dense networks, energy management, cell management 


\section{Introduction}

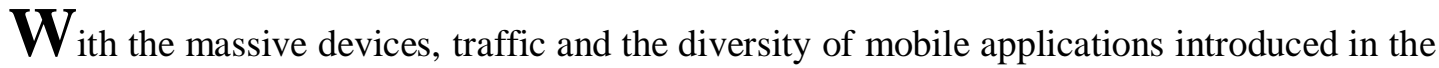
mobile heterogeneous networks, the big data era for mobile internet is approaching[1][2]. There is the need to efficiently manage all the resources involved in the Mobile Internet. Massive densification of Radio Access Network (RAN) infrastructure known as Ultra Dense Network (UDN) is considered a promising solution to meet the rapidly increasing demand for radio access networks. UDN is proposed to further improve throughput per user and to meet the capacity requirement in peak hour per square meters [3]. The escalating data traffic volume and the dramatic expansion of the network infrastructure will inevitably trigger an increased energy consumption in future wireless networks. This will directly increase the greenhouse gas emissions and mandate an ever increasing attention to the protection of the environment. Consequently, both industry and academia are engaged in working towards enhancing the network energy efficiency.

Maximizing the network energy efficiency may be supported by maximizing the amount of throughput, while minimizing the total energy consumption. As far as the problem formulation is concerned, maximizing the network energy efficiency can be expressed as minimizing the total energy consumption while satisfying the associated traffic demands. For example, in the valley of traffic, huge energy consumption by ultra-dense radio access network has become an economic and environmental concern to network operators. However, redundancy of radio resource was not exploited when user association relationship can be rechanged in ultra-dense network. Hence, the network energy efficiency is crucially dependent on the user association decisions [4]. In solving problems with the energy consumption of ultra-dense radio access network, several studies in recent time suggested the scheme, which is known as Multiple Base Station Scheduling(MBSS) [5][6][7][8]. MBSS leads to the substantial energy efficiency gains in the LTE-compliant wireless network deployments. However, the main problem with MBSS is its computational complexity[9]. With the increasing dimension of ultra-dense nodes, the computational complexity will affect the effective practical implementation of the energy saving algorithm. Moreover, the mobility of user, and the traffic dynamics compound the computational problems associated with MBSS. An approach, flow Scheduling, is therefore necessary not only to reduce the energy consumption but to take into account user mobility and traffic dynamics within ultra-dense networks.

From the perspective of radio resource management, it is proposed in this paper, how efficient energy consumption adapts to the density of traffic. This is achieved through the proper scheduling of the flows over BS in dense small cell deployment. We proposed an energy manager, which is a part of soft-RAN architecture. The architecture includes a switch-on/off control algorithm by scheduling flows to reduce the number of active BSs based on traffic dynamics, which considers the quality of real-time services.

The organization of the rest of this paper is as follows. Section II presents related works and how differently our approach is. Section III presents the proposed soft-RAN architecture necessary for the reduction in energy consumption. Section IV deals with the energy management scheme whiles section $\mathrm{V}$ presents the performance evaluation of the proposed scheme. The summary of the contributions of this paper and conclusion are presented in section VI. 


\section{Related Work}

Numerous valuable contributions have been published on energy efficient user association in HetNets [10], [11], [12], [13], [14]. In [10], a user association algorithm was developed for the uplink of HetNets in order to maximize the system energy efficiency subject to users' maximum transmit power and minimum rate constraints. In [13], user association for the downlink of HetNets was optimized by maximizing the ratio between the total data rate of all users and the total energy consumption. In contrast to the problem formulation in [10], the authors investigated energy efficient user association in [11] by minimizing the total power consumption, while satisfying the users' traffic demand. The authors of [12] considered the association problem for users involving video applications, where a video content aware energy efficient user association algorithm was proposed for the downlink of HetNets, with the goal to maximize the ratio between the peak-signal-to-noise-ratio and the system energy consumption. Thereby, both nonlinear fractional programming and dual decomposition techniques were employed to solve the problem. In [14], a Benders' decomposition [15] based algorithm was developed for joint user association and power control with the goal of maximizing the downlink throughput. This was achieved by associating every user with the specific BS, which resulted in the minimization of the total transmit power consumption.

Previously, energy consumption of BS equipment has been intensively investigated, mainly concerning the consumption by devices and supporting system, e.g. power supply and cooling system. Statistical studies of mobile communication systems have shown that $57 \%$ of the total energy consumption of wireless networks can be attributed to the radio access nodes [16]. Furthermore, about $60 \%$ of the power dissipated at each BS is consumed by the signal processing circuits and air conditioning [17]. Data from manufacturers indicates that.energy consumed for radio transmission only constitutes a small fraction of total energy consumption. As a result, shutting down BSs which support no active users is believed to be an efficient way of reducing the network power consumption [18], [19]. Hence, a feasible approach to improve energy efficiency is switching off BSs rather than merely adjusting transmitting power.

Lately, the analysis from the perspective of whole network, by switching off fraction of BSs in low traffic period (night zone) is introduced [20][21][22][23]. A joint optimization of the long-term BS sleep-mode operation, user association, and subcarrier allocation was considered in [20], for maximizing the energy efficiency or minimizing the total power consumption under the constraints of maintaining an average sum rate target and rate fairness. The performance of these two formulations (namely, energy efficiency maximization and total power minimization) was investigated using simulations. In addition, the coverage probability and the energy efficiency of K-tier heterogeneous wireless networks were derived in [21] under different sleep-mode operations using a stochastic geometry based model. The authors formulated both power consumption minimization as well as energy efficiency maximization problems and determined the optimal operating regimes of the macrocell. In [22], the discussion is extended with real traffic profile and dynamic control. In previous work done in operational stage, the available configurations of cells could not be changed. This has assumed that sufficient cell zooming could provide service for area originally served by cells that are switched off. However, insufficient cell zooming that prevents switching off more cells was not considered. To combat insufficient cell zooming, little work has been done to discuss whether it is energy-efficient to adjust the deployment in the network planning stage. One feasible solution is to deploy smaller but more cells [23]. However, the real traffic is often much lower than the peak-hour traffic and certain small cells are superfluous. If we switch off certain cells only through cell zooming, energy saving would be limited. This paper, on the 
other hand, proposed an elastic energy efficient cell management scheme based on short-term flow scheduling among multi-layer cells by a SDN controller.

\section{System architecture and problem description}

Here, a UDN environment is considered, where small cells are overlaid by macro cells. The set of BSs can be turned on/off based on the statistics and characteristics of flows associated with them by a cell-level energy manager. The BSs which are turned on are referred as active BSs and the BSs which are turned off, are referred as inactive BSs. In Fig. 1, we define an architecture of software defined UDN.

- Monitoring Module: With the User Equipment (UE) measurement report messages, the Openflow controller obtains knowledge about the status of every small cell BS. The Openflow controller manages small cells, either in active or in sleep mode. The traffic over this UDN can be divided into two types of flows, real-time and non-real time. The flow scheduling over different BSs is defined as association or handover operation. A handover occurs during the moving of ongoing call or data session from one BS to another. We assume the real-time flow is sensitive to handover intermittent gap, so we need to avoid to handover the real-time flows for energy saving. If there is sufficient bandwidth available, the flows can be aggregated over a minimized BS set in order to reduce the energy consumption.

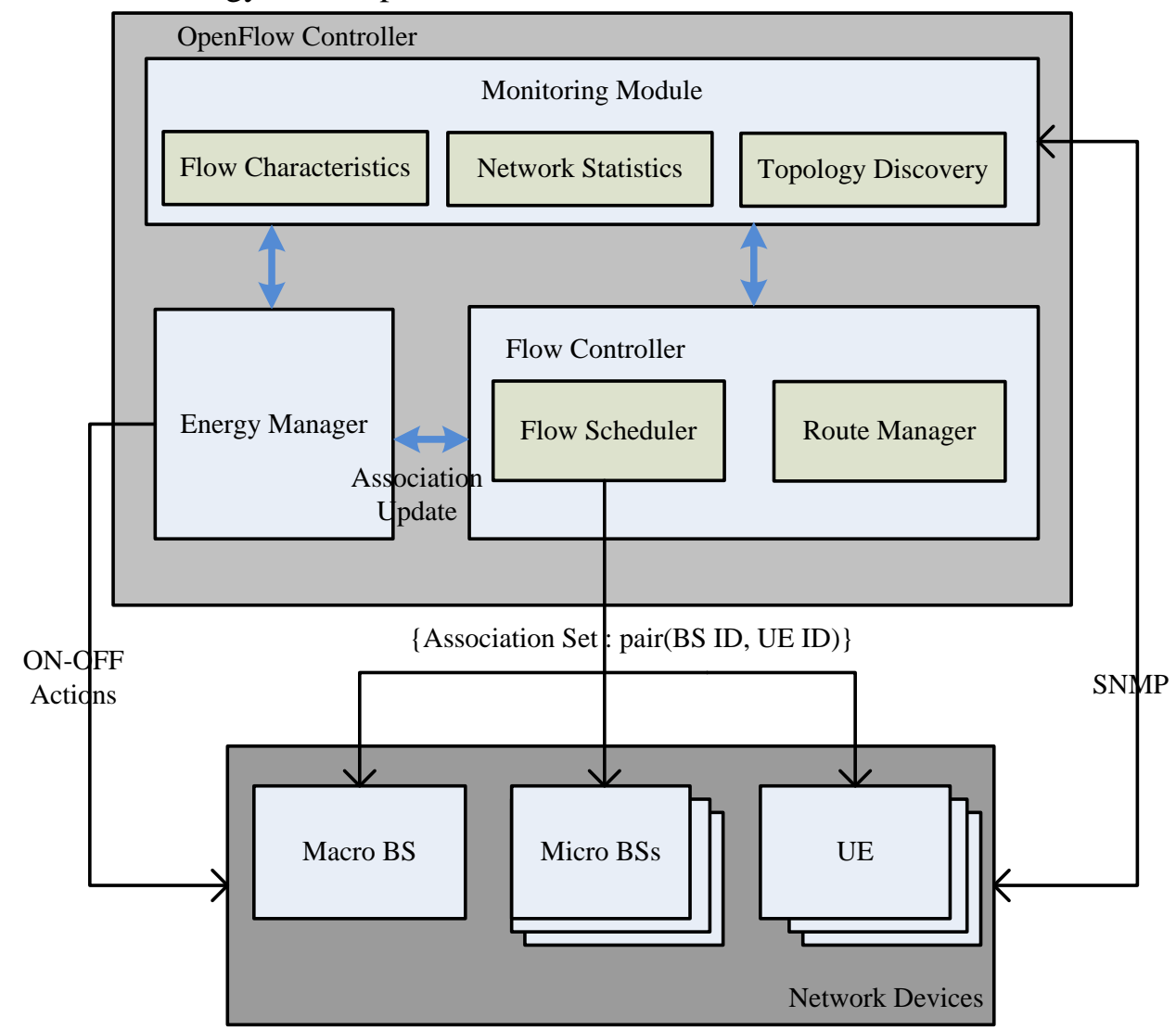

Fig. 1. Architecture of the centralized energy management 
- Energy Manager : The energy manager controls switches of BSs in a cell level via control plane. The energy manager can turn on a sleep BS, if some real-time flow is located inside its coverage. There is a signaling interface on the control plane for the network access and a message definition between the flow controller and the energy manager, shown in the Fig. 1. In order to reduce energy consumption, the energy manager scans each active BS in a fixed period, e.g. Half-an-hour, and sends a switch-off command to the idle BSs. A BS will enter sleep mode, if there is no transmit or receive command from its neighbored UEs. The scanning period can be configured by the controller via SSL.

- Flow Controller : The flow controller manages the flows over a set of BSs on data plane under the controller. It includes route manager for wired network and the proposed flow scheduler in radio access network.

- The route manager will help new flows find their best next hop to the destination based on the topology information and new flow request. The updated association relationship will be sent to energy manager to uptate BS status and notified to BS and UE to execute the association change.

- The proposed flow scheduler dynamically associates the flows over the BSs based on the characteristics of the traffic, as shown in the Fig. 2. Based on the QoS requirement of traffic, flows can be divided into real-time and non-real-time flows. With the traffic profiles on the other hand, flows can be divided into four kinds in terms of the packet size and the duration gap, with different amount of bandwidth. Based on power status, the BS can be divided into three sets: active, idle, sleep. With respect to coverage, cells are divided into macro, and micro/small cells.

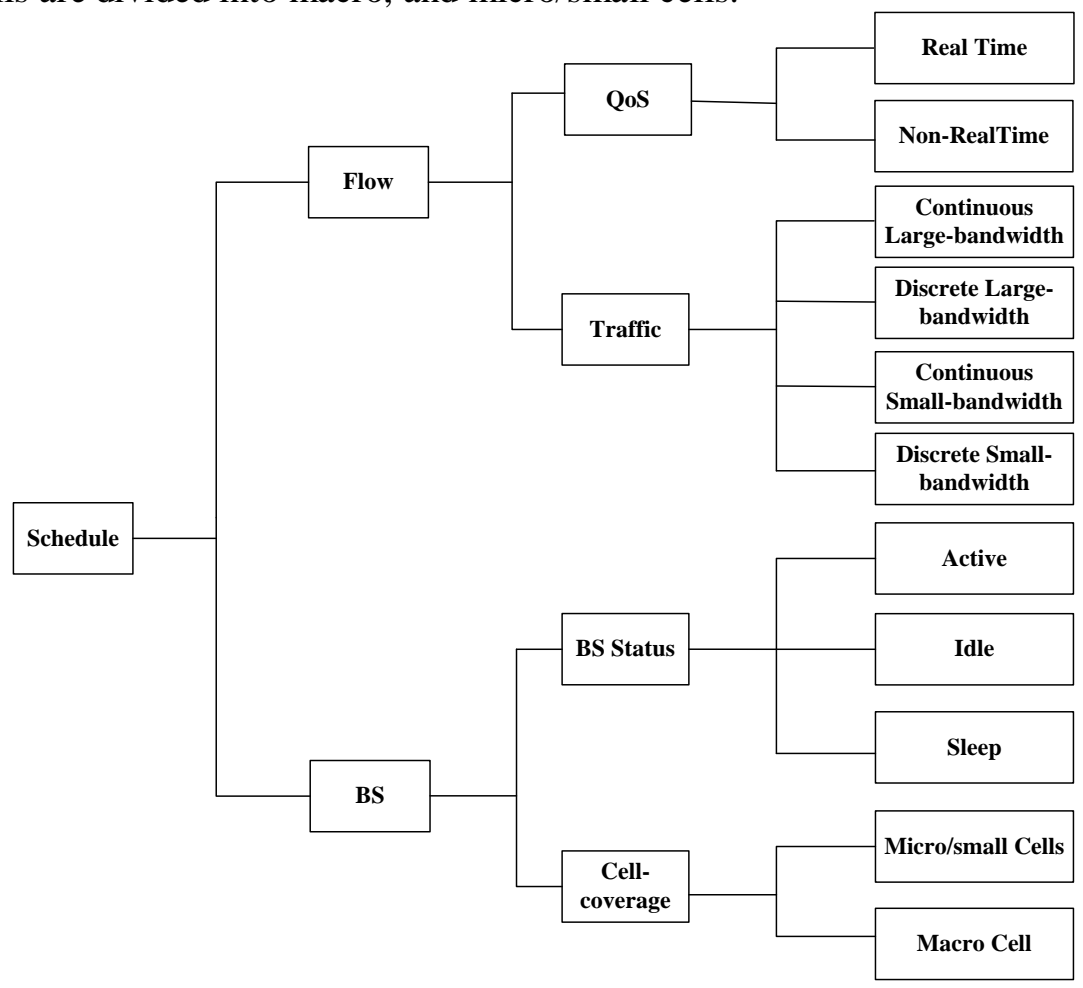

Fig. 2. The characteristics classification for scheduler 


\section{The Proposed Algorithm}

\subsection{The energy consumption model}

In this paper, we propose an energy management algorithm based on flow scheduling and cell switching on/off as defined in Fig. 3. The algorithm switches on BSs in sleeping mode when a new flow enters into the cell, and switches off active BSs when no flows enters the BS or when the flows can be scheduled onto a neighboring small cell. Cells need to change their on-off states by the scheduling scheme on the controller. Different scheduling schemes will lead to different energy consumption. We can define the different scheduling schemes with parameters for a single small cell. The total power consumption is $P$. The $P(j)$ is the power consumption of the $j^{\text {th }}$ active cell, and $i$ represents scan cycle for one day. Given the traffic and bandwidth constraint, we shall minimize the accumulated energy consumption of network $E(i)$ for each cycle $i$ in one duration $T$.

$$
\begin{aligned}
& \min E=\int T P(t) d t=\sum_{i} \sum_{j} P(j) C \\
& \text { s.t } \sum_{j} f_{j}(i)<f_{\text {threshold }} \\
& \quad b_{j}(i)<b_{\text {max }} \\
& 0<i<\frac{\mathrm{T}}{\mathrm{C}}, \quad 0<j<N
\end{aligned}
$$

Where $C$ is a scan cycle, $P(t)$ is the total power of network changing with time $t$. $f_{j}(i)$ is the number of flow which would be scheduled over the BS $\mathrm{j}$ in the $i^{\text {th }}$ scan cycle. The $f_{\text {threshold }}$ is the maximal number of flow which can be scheduled in every scan cycle. The value of $f_{\text {threshold }}$ is configured on the controller. $b_{j}(i)$ s the occupied bandwidth of the BS $\mathrm{j}$ in the $i^{\text {th }}$ scan cycle. The $b_{\max }$ is the threshold of bandwidth of one small BS. $T / C$ is the number of scan cycle of one day.

Definition 1: Based on the characteristics of flow and cell, the scheduling cost of the BS $j$ to be switched off in the $i^{\text {th }}$ scan cycle, is defined as $\operatorname{Cost}_{j}(i)$ :

$$
\operatorname{Cost}_{j}(i)=f_{j}(i) \times b_{j}(i)
$$

We can sort the active BS set based on the value of $\operatorname{Cost}_{j}(i)$. Based on this tool function of scheduling cost, a heuristic algorithm is proposed as the flow and cell scheduling schemes according to this sequence.

\subsection{The proposed algorithm}

The proposed algorithm determines which active BS can be switched off, and which inactive BS can be switched on to update its original membership. The proposed algorithm for the unified energy manager is given in Fig. 3. Because incoming UEs with real-time flow can be in the range of an active or inactive BS, it leads to handover flows from one BS to the other. We assume each UE maintains a list of neighboring cells, which is the candidate for cell association. The BS scheduler is triggered by timer, e.g. half-an-hour.

The switch-on scanner starts its work by getting flow ID and uses this flow ID to get the characteristics of the flow, such as the location of the UE and the neighboring BS ID. It then checks if the neighboring BS is active, if so, it connects this flow to that BS. If the neighboring 
BS is inactive it checks whether the current flow is real time flow or not, if this flow is non real-time, it checks if there is enough bandwidth available on the Macro BS to support the current flow. If so it connects the current flow to the Macro BS, else it will switch on the neighboring BS. Finally it checks if there are non-real-time flows in the Macro BS that can be supported by recently switched on neighboring BS, if so, it handovers those flows to this BS. The BS set scanner targets to execute the switch-off actions when the BS is idle, as illustrated in the Fig. 3. The active BS set includes all BS associated with UEs. The switch-off arbitration begins its procedure by scanning the active BS set. If the BS is associated with real-time flows, it keeps this BS on, then checks the next active BS. Else, it computes the cost for each BS, used to identify traffic load. If the sum of bandwidth of flows associated with this active BS is less than the available bandwidth in the macro BS, flow handovers will happen from small cell to macro cell, which is also known as flow handover. Then this active BS is switched off. It then continues to check the next active BS in the waiting list. This procedure works in a periodical manner. The status of BS leads to different amount of energy consumption.

\section{THE PROPOSED ALGORITHM}

Notations: NBS: Neighbored BS; MBS: Macro-BS; ABSS: Active BS Set; IBSS: In-active BS Set; MCBS: Minimum Cost BS

Phase I - When a new incoming flow access $B S$

1 select the nearest $B S$ as NBS;

2 if(NBS is Active)

new flow access NBS;

$3 \quad$ elseif(new flow is non-real-time flow \&\& free bandwidth on

$4 \quad M B S>$ new flow's bandwidth requirement)

$5 \quad$ else

6 new flow access $M B S$;

Switch on NBS and add it into ABSS;

7 for (flow in $^{\mathrm{M}} \mathrm{MBS}$ )

8 if $\left(\right.$ flow $_{i}$ reachable to NBS)

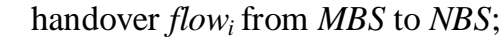

9

10

11

end if

end for

end if

Output: whether to handover flow;

Phase II - When it's time to finish each scanning cycle

$1 \quad$ for $\left(B S_{j}\right.$ in $\left.A B S S\right)$

$2 \quad$ if $\left(B S_{j}\right.$ has realtime flow)

keep $B S_{j}$ active and don't handover;

3 else

$4 \quad$ calculate cost $t_{j}=f_{j} \times b_{j}$;

$5 \quad$ end if

6 end for

7 if(occupied bandwidth on $M C B S<M B S$ 's residual bandwidth and

8 the number of flows on $M B S<f_{\text {threshold }}$ )

handover all of flows on MCBS to MBS and shut-down MCBS.

9 update $A B S S \& \&$ IBSS with MCBS;

10 end if

11 Output: ABSS\&\&IBBS

Fig. 3. The proposed algorithm for energy efficient cell management 


\section{Results and Analysis}

The proposed energy efficient cell management algorithm is evaluated with the idealized traffic profiles and the real traffic profiles. The evaluation is performed in a scenario with 110 small cells and one macro cell. The inter-site distance between the small cell BSs is $50 \mathrm{~m}$. The UE distribution is uniform in this area. The used metrics are the total energy consumption per time cycle, the number of active BSs, the number of handovers, the number of switch-off/on, and the time duration of sleep/idle/active. In the simulation, the BS schedule period is configured as 30 minutes respectively for the purpose of this experiment.

\subsection{Daily Traffic configuration}

The distribution of UE and UE's traffic are modeled by a daily traffic model, in which the number of active UEs, and traffic demands vary over the clock time in a day. A traffic profile is based on the real measurements from the EARTH project [24], as illustrated with case I in the Fig. 4. The daily data traffic profile in one hour average, which has to be served typically by a single operator in Europe for beyond 2015 for dense urban areas. Thereby, we illustrate the activities of incoming users with case II and the leaving users with case III in the Fig. 4.

An ideal traffic profile is configured based on the trapezoidal traffic pattern, which is a special simple example of daily traffic pattern for the family of symmetric trapezoidal curves plotted in the Fig. 4. The traffic function is defined by the angular coefficient $a$, which has a maximum equal to 1 at the peak hour, and different slopes.

$$
f(t)=\left\{\begin{array}{c}
1-a t ;(0 \leq t<1 / a) \\
0 ; \\
1+a(t-T) ;(T-1 / a \leq t \leq T)
\end{array}\right.
$$

If " $a$ " is equal to $1 / 10$, then we move the $f(t)$ to $f(t)+12$, which is close to the real scenario, as shown with case IV in the Fig. 4. If the slope " $a$ " is equal to $1 / 8$, the traffic profile will be changed, as shown with case III in the Fig. 4 to mimic the variant traffic profile in different spatial-time domain. Then, we consider the impact of traffic profiles on energy saving.

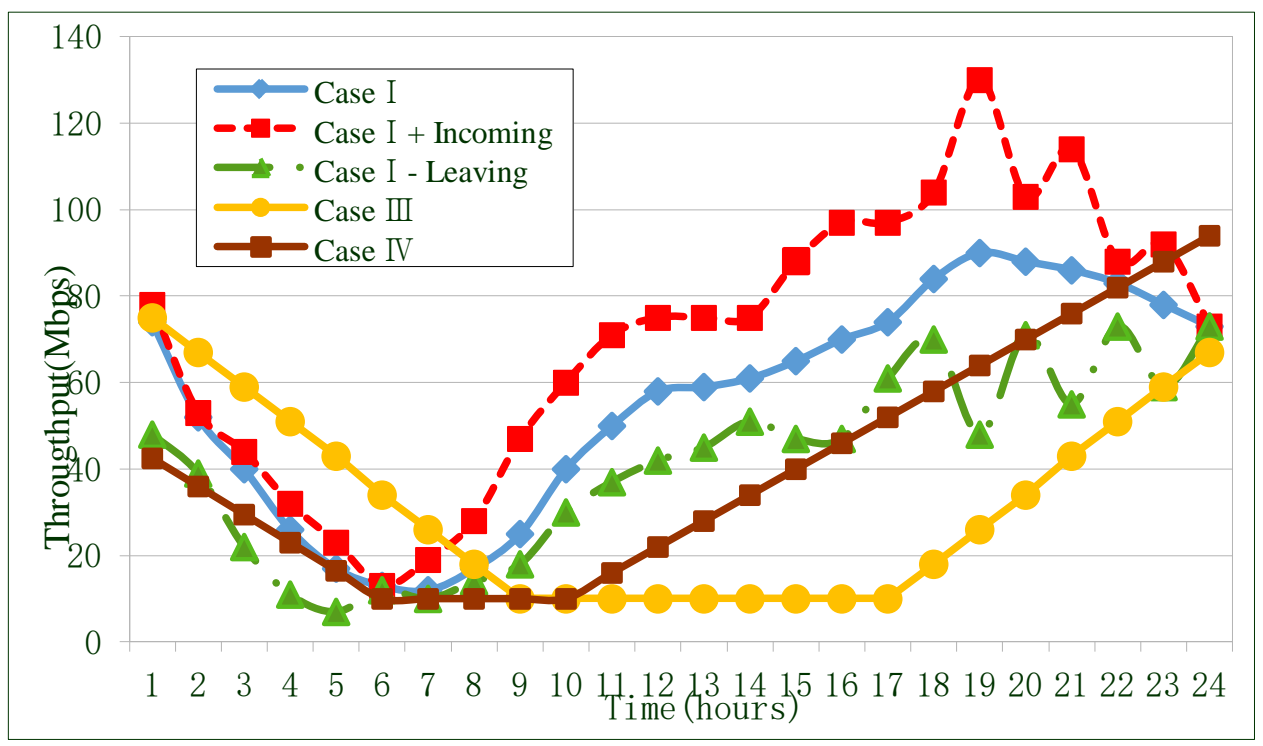

Fig. 4. The traffic profile configuration: Ideal model and Real traffic 
Here, we assume each UE is configured with one flow, with similar bandwidth, e.g. $1 \mathrm{MHz}$, so the number of flow is proportion to the throughput. In the Fig. 4, the highest average throughput of $92 \mathrm{Mbps} / \mathrm{km}^{2}$ and the lowest of $12 \mathrm{Mbps} / \mathrm{km}^{2}$ can be observed for the dense urban area in the late evening. In the ideal traffic profile, the slope parameter $a$ can be tuned, e.g. 1/8, $1 / 10$ and so on in our test cases. The flows and UEs join the network in a dynamic manner. The activities of flows and UEs, leaving or coming, are recorded with (Case I-Leaving) and (Case+ Incoming) respectively in dash lines, as shown in the Fig. 4. Energy consumption is changing with the flow activities. The number of inbound/ outbound flows is controlled in dash lines proportionally.

\subsection{Energy consumption in dynamic scenario}

To illustrate the achieved energy consumption of the proposed algorithm, a simulation is done with three traffic profiles for the proposed algorithm. The total energy consumption with three traffic profiles is compared: two idealized traffic profiles with $a=1 / 8$ (Case III) and $a=1 / 10$ (Case IV), one common real traffic profile(Case I). The results show that the performance of the proposed algorithm with Case I, III and IV outperforms the direct On-Off scheme with Case II, which is called MBSS[9]. The results are shown in the Fig. $\mathbf{5}$.

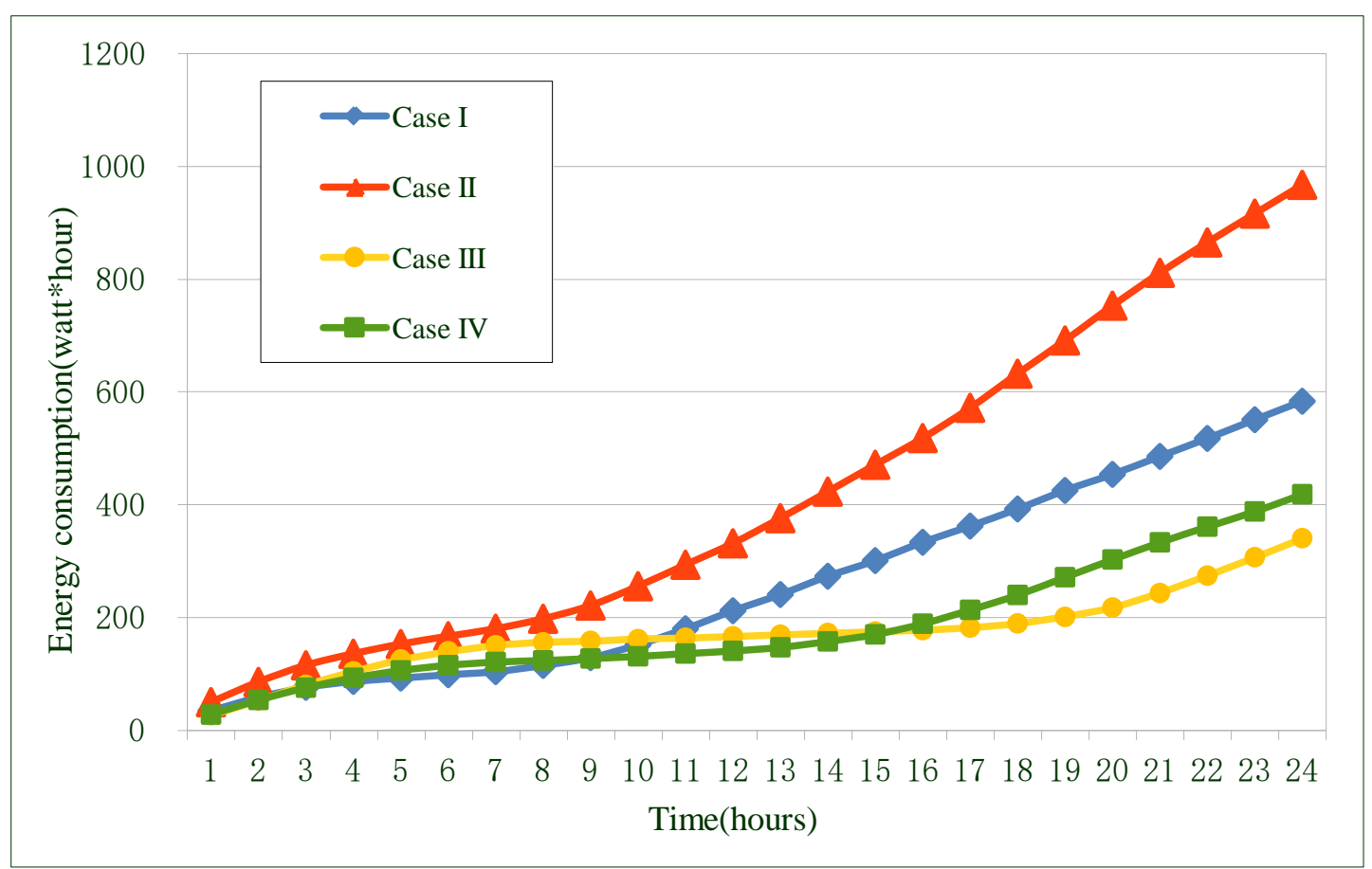

Fig. 5. The total energy consumption with different traffic profiles

\subsection{The number of active BSs}

The flow activity leads to the activities of BS in active, idle or sleep. The number of active BS in our case is illustrated in the Fig. 6. The results show that the number of profiles of active BSs is proportional to the volume of traffic. For the real traffic profile in Case I, and the ideal traffic profiles in Case III and IV, the number of active BSs with the proposed algorithm is less than the MBSS with direct power on/off in Case II . 


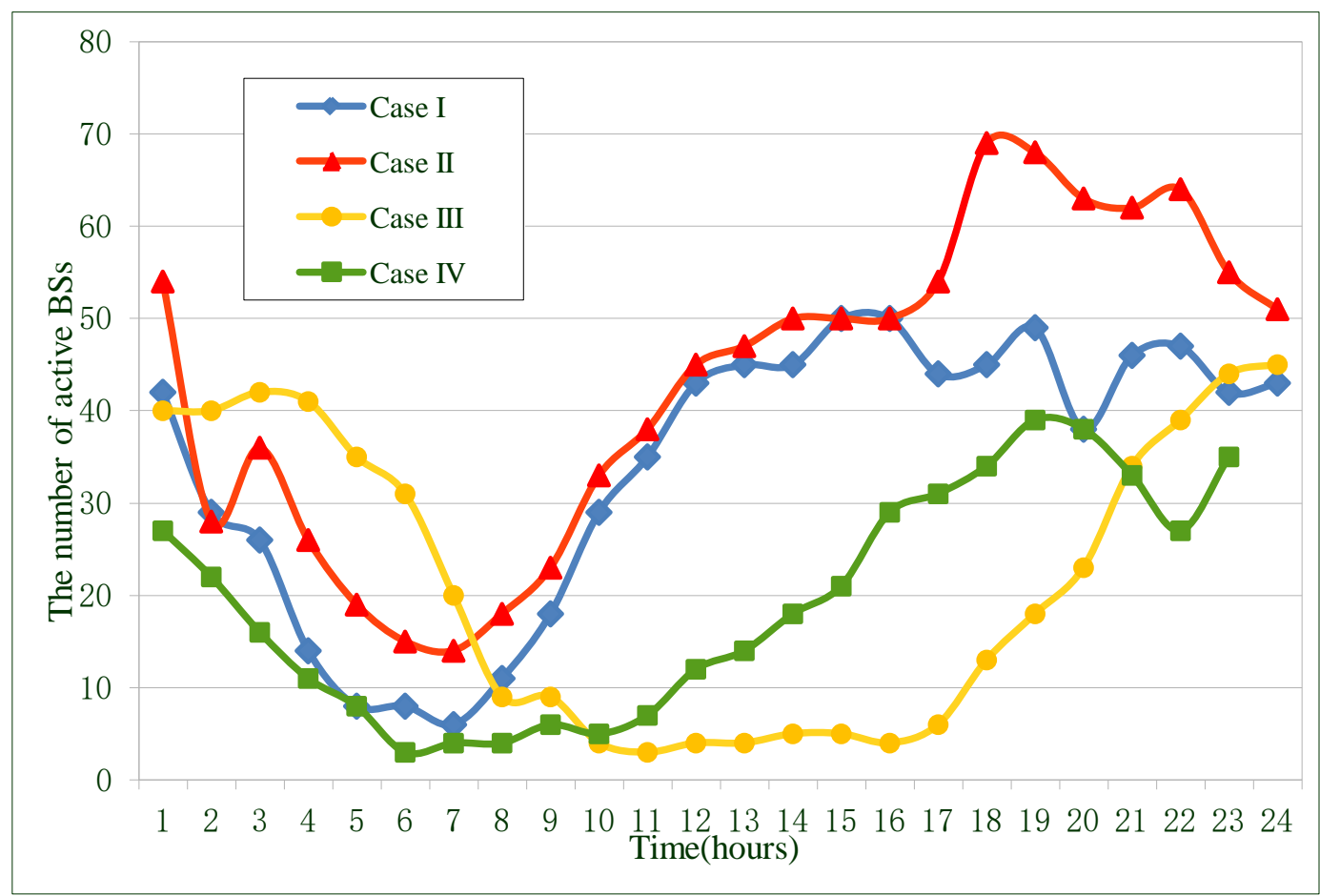

Fig. 6. The number of active BSs in each period during 24hours

\subsection{The number of flow handovers}

Flow handover could happen in each scanning period. The total number of flow handover is similar and proportionate to the traffic dynamics with the exception of the incoming real-time flow, whatever the real traffic profile in Case I for the proposed algorithm and Case II for the direct On-Off scheme, and the ideal traffic profiles in Case III and IV. This is shown in the Fig. 7.

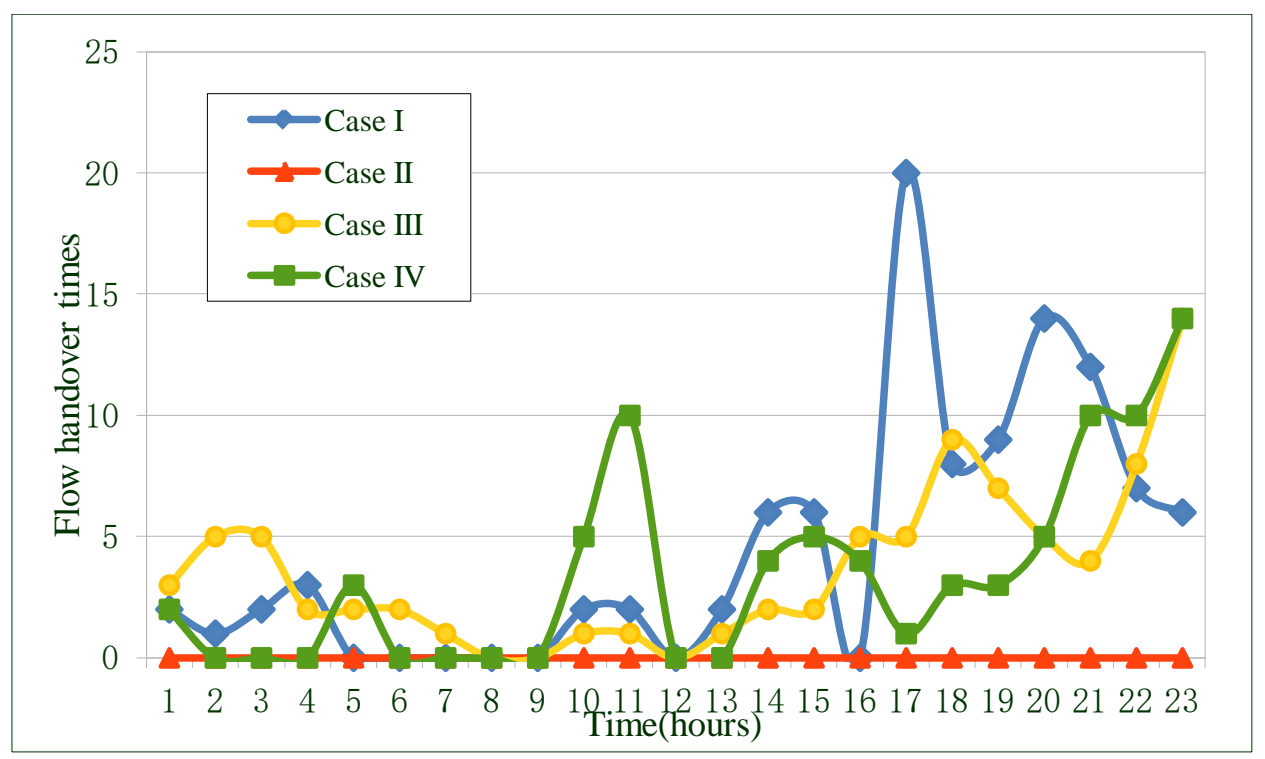

Fig. 7. The number of flow handover in each period 
Fig. 8 shows that, the proportion of incoming real-time flows has the effect on the number of flow handover. During a whole day in 24 hours, we configure the ratio of incoming non-real time flow amount and the real-time flows amount as $1,1 / 3$ and 0 respectively. The results show the handover times increases with more of non-real-time flows coming.

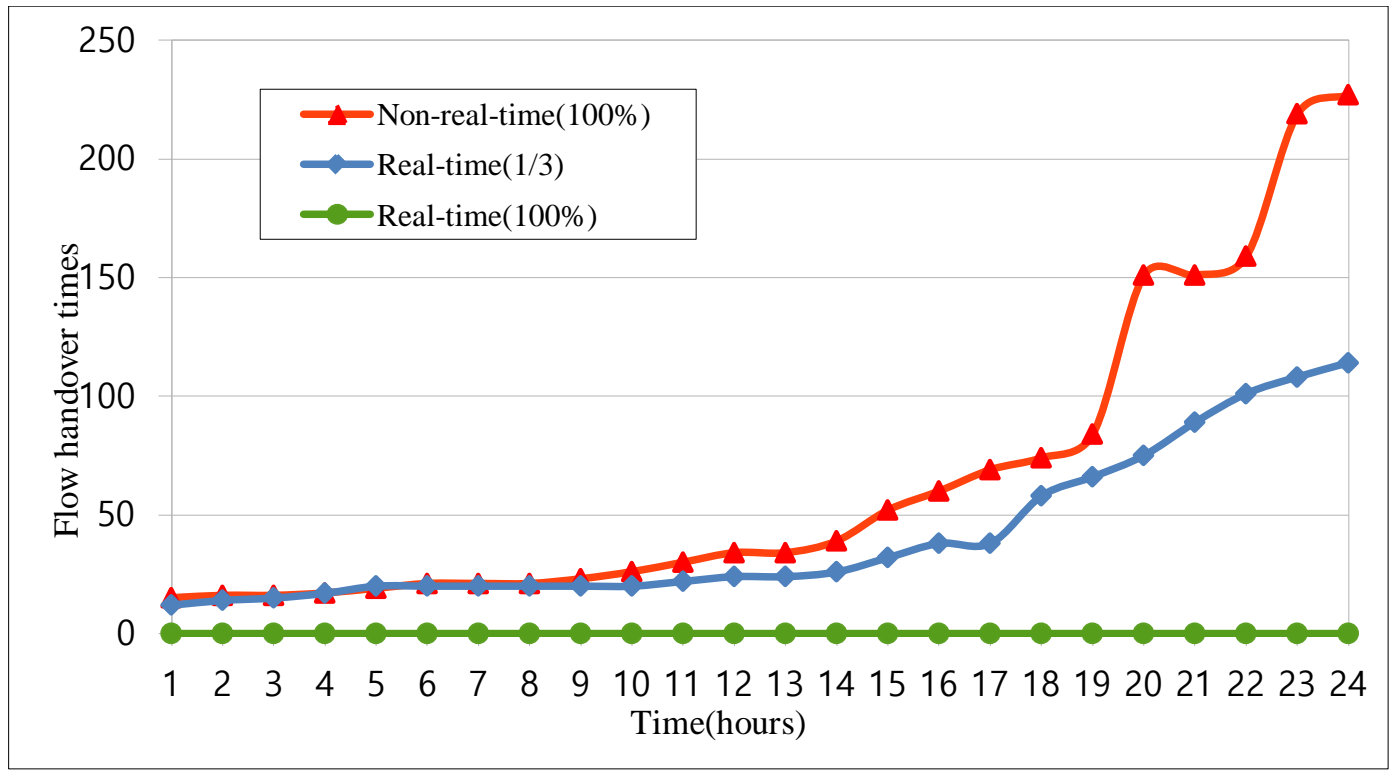

Fig. 8. The total number of handover for hybrid flows

\subsection{The number of BS switches}

The BSs can be switched on or off in each BS scanning period. The number of BS switches is recorded in each period. For the real traffic profile in Case I, and the ideal traffic profiles in Case III and IV, the proposed flow scheduler performs better than the direct On-Off scheme with the real traffic in Case II. The results are shown in the Fig. 9.

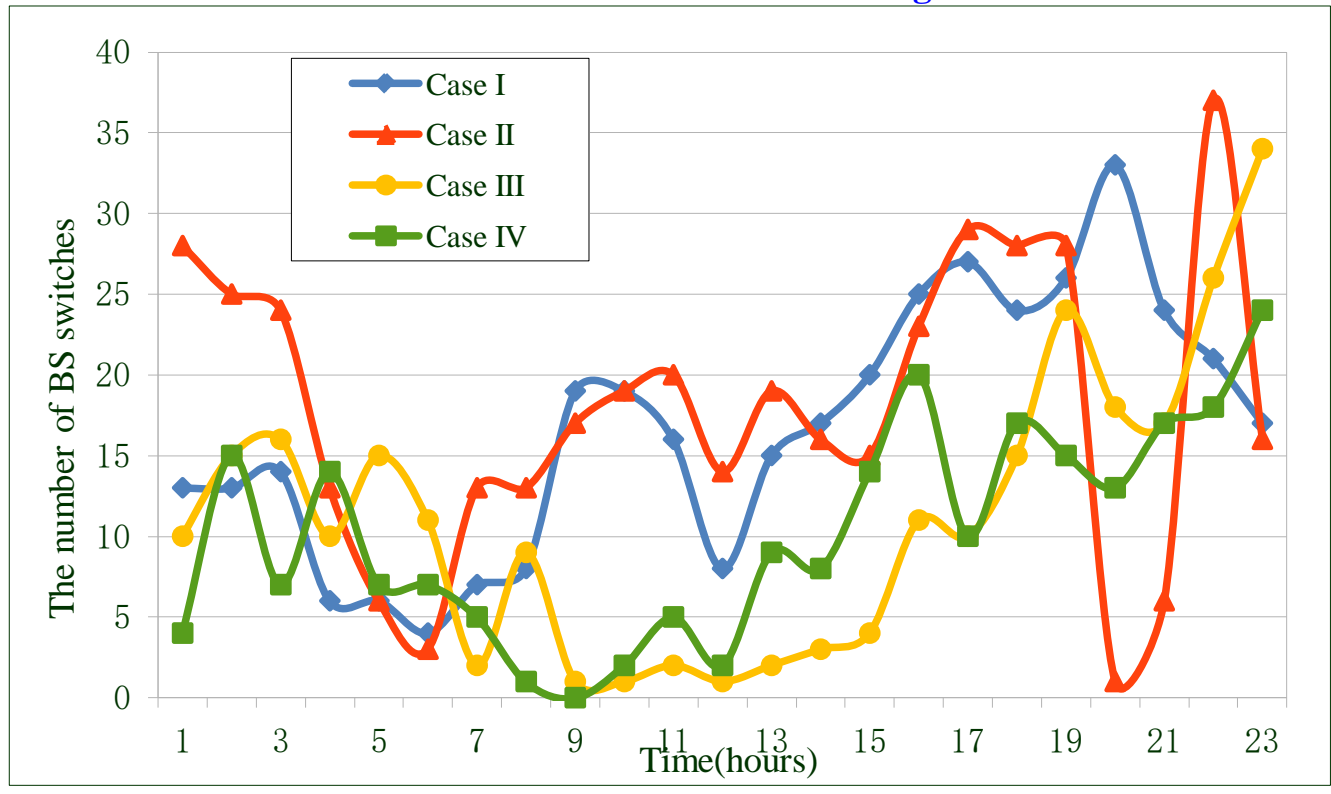

Fig. 9. The number of BS switches in each period 


\subsection{Sleep/ldle/Active Time Duration}

The energy consumption mostly depends on the transmitter power setting of each BS. The transmitter power of BSs is set as $30 \mathrm{dBm}, 10 \mathrm{dBm}$ and $0 \mathrm{dBm}$ respectively for the active, idle and sleep BSs. The durations in active, idle and sleep mode for all of BSs in whole a day are recorded and illustrated in the Fig. 10(a), (b) and (c) respectively. The sum of idle, active, sleep duration of BSs for one common traffic profile in each period is a constant.

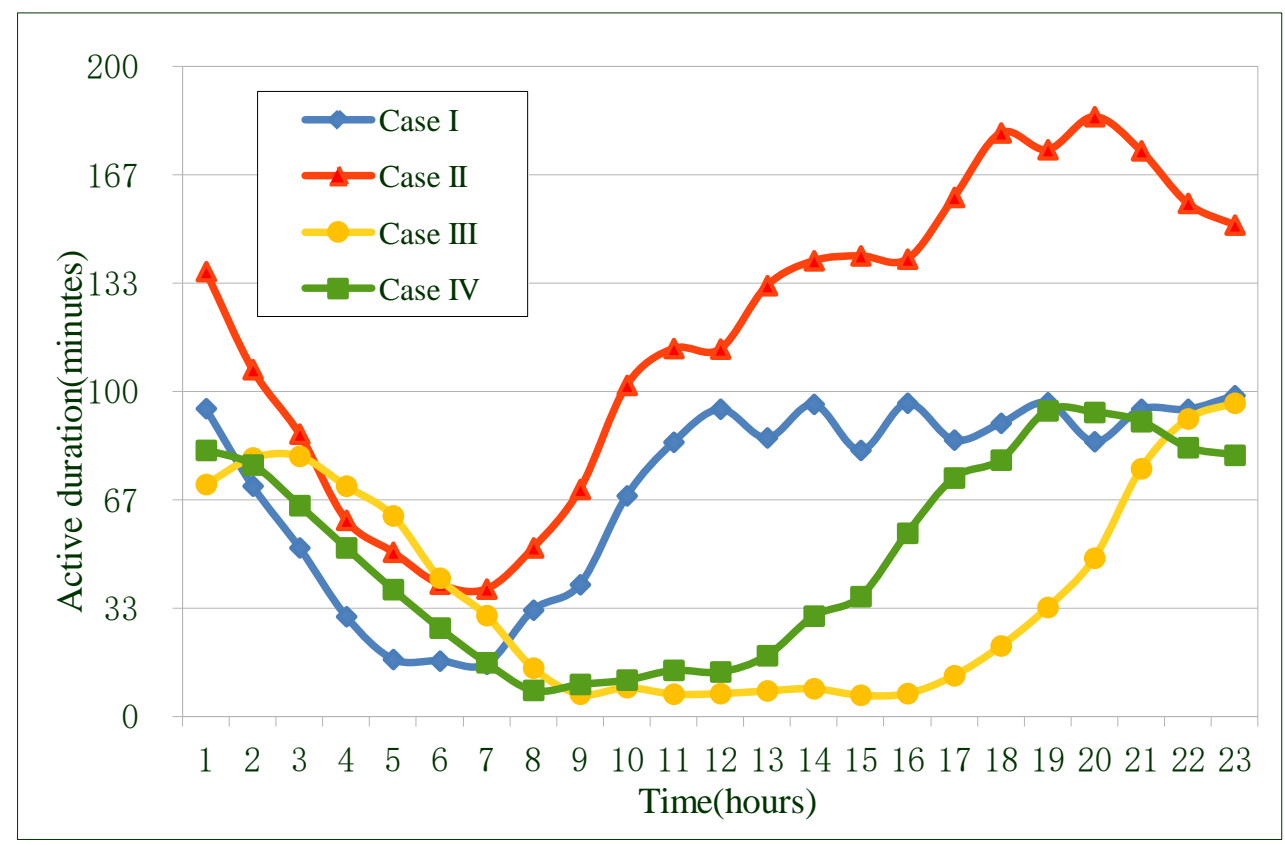

(a)

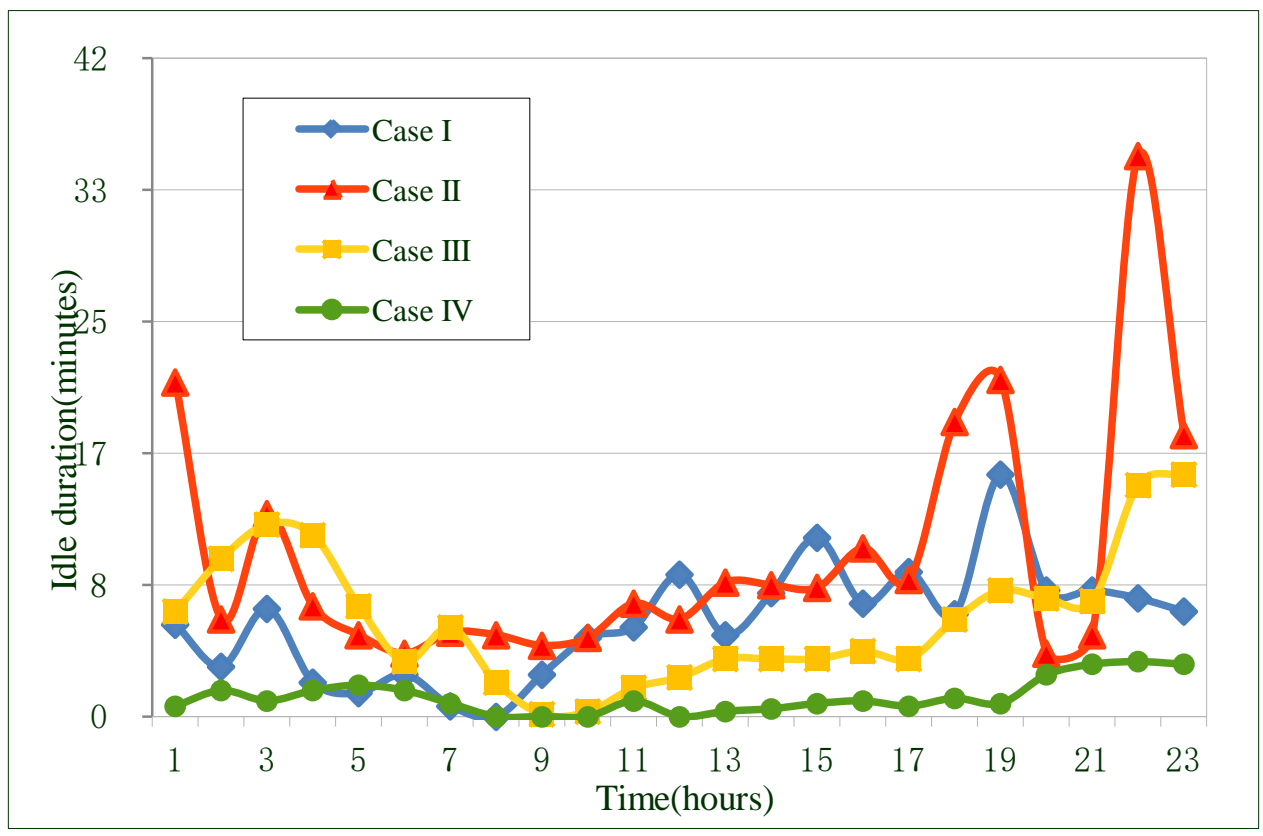

(b) 


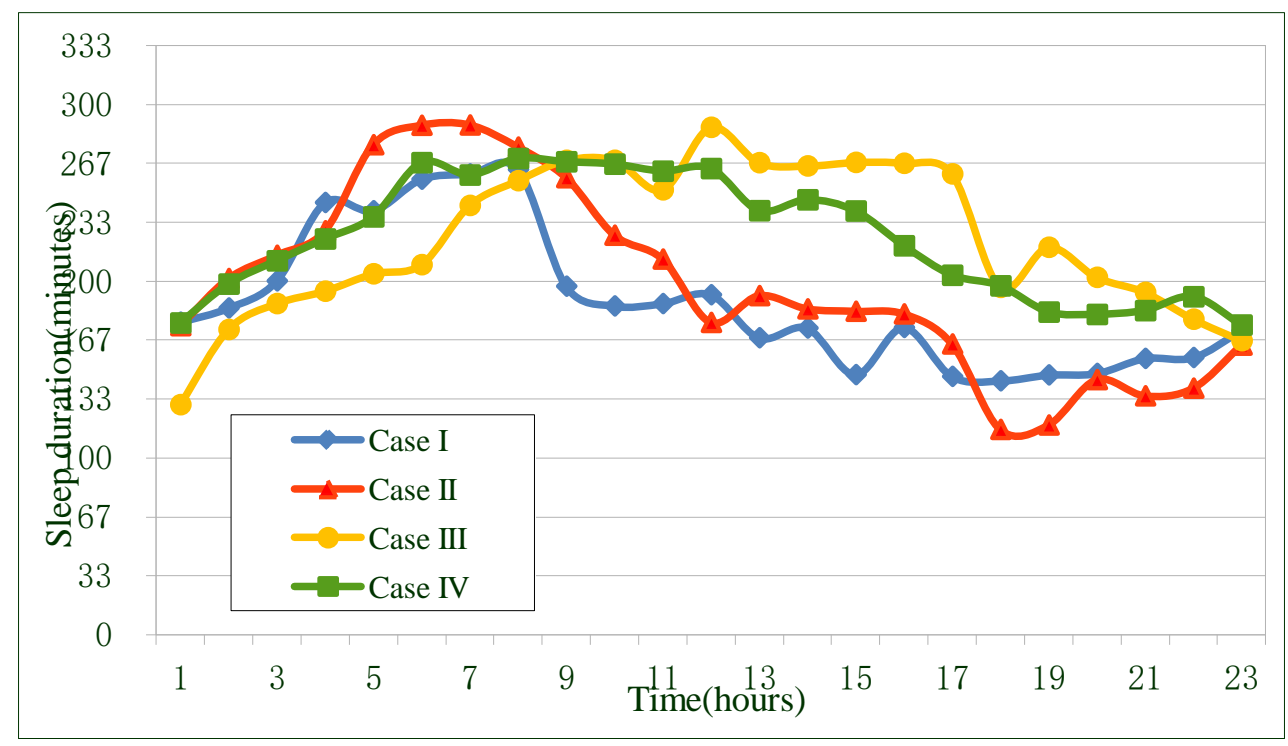

(c)

Fig. 10. The active/idle/sleep duration in each period

\section{Conclusion}

In this paper, an elastic energy efficient cell management scheme is proposed by properly scheduling BS switch-on/off in order to balance energy consumption and the quality of service guarantee. Unlike previous studies characterized by computational complexity and inadequate provision for user mobility, this paper proposed a more dynamic approach. The proposed energy management algorithm is based on the flow and BS scheduling. The flow scheduler will hand over some of flows based on the traffic dynamics and flow activities. The target is to aggregate flows to a minimized active BS set. The experiment results show the algorithm performs better on the number of active BS, the number of BS switch on/off and the number of handovers than previous ones. This inevitably reduced the energy consumption of each BS.

\section{Acknowledgment}

This work is supported by the Specialized Research Fund for the Doctoral Program of Higher Education of China, Grant no. 20130185120021, by the Fundamental Research Funds for the Central Universities under grant no. ZYGX2014J060, and by the ZTE Innovation Research Fund for Universities Program 2014 under grant no. CON1409180014.

\section{References}

[1] Minho Jo, Taras Maksymyuk, Bohdan Strykhalyuk, and Choong-Ho Cho, "Device-to-device-based heterogeneous radio access network architecture for mobile cloud computing," in Proc. of IEEE Wireless Communications, vol. 22, no. 3, pp. 50-58, Jun. 2015. Article (CrossRef Link)

[2] Minho Jo, Taras Maksymyuk, Rodrigo L. Batista, Tarcisio F. Maciel, André L.F. De Almeida, and Mykhailo Klymash, "A survey of converging solutions for heterogeneous mobile networks," in 
Proc. of IEEE Wireless Communications, vol. 21, no. 6, pp. 54-62, Dec. 2014. Article (CrossRef Link)

[3] Bhushan, N., Junyi Li; Malladi, D., Gilmore, R., Brenner, D., Damnjanovic, A., Sukhavasi, R., Patel, C., Geirhofer, S., "Network densification: the dominant theme for wireless evolution into 5G," IEEE Communications Magazine, vol.52, no.2, pp.82-89, Feb. 2014. Article (CrossRefLink)

[4] A. Mesodiakaki, F. Adelantado, L. Alonso, and C. Verikoukis, "Energy efficient user association in cognitive heterogeneous networks," IEEE Commun. Mag., vol. 52, no. 7, pp. 22-29, Jul. 2014. Article (CrossRef Link)

[5] Koudouridis, G.P., Hui Gao, Legg, P., "A centralised approach to power on-off optimisation for heterogeneous networks," in Proc. of 2012 IEEE Vehicular Technology Conference (VTC Fall), vol.1 no.5, pp. 3-6, Sept. 2012. Article (CrossRef Link)

[6] Falconetti, Laetitia, Frenger, Pal, Kallin, Harald, Rimhagen, Thomas, "Energy efficiency in heterogeneous networks," in Proc. of IEEE Online Conference on Green Communications (GreenCom2012), vol.98, no.103, pp. 25-28, Sept. 2012. Article (CrossRef Link)

[7] Amerga, Messay, Bhupesh Umatt, and Vineet Mittal, "Sleep mode for wireless communication device during out of service operation," U.S. Patent 8,527,014, Sept. 2013. Article (CrossRefLink)

[8] Ashraf, Imran, Lester TW Ho, and Holger Claussen, "Improving energy efficiency of femtocell base stations via user activity detection," in Proc. of IEEE Wireless Communications and Networking Conference (WCNC) 2010, pp.1-5, Apr. 2010. Article (CrossRef Link)

[9] Zhou, Chan, and Omer Bulakci, "Stability-aware and Energy Efficient Cell Management in Ultra Dense Networks," in Proc. of 2014 IEEE 80th Vehicular Technology Conference (VTC Fall), pp.1-5, Sept. 2014. Article (CrossRef Link)

[10] Mesodiakaki, F. Adelantado, L. Alonso, and C. Verikoukis, "Energy efficient context-aware user association for outdoor small cell heterogeneous networks," in Proc. of IEEE Int. Conf. Commun. (ICC), pp. 1614-1619, Jun. 2014. Article (CrossRef Link)

[11] H. Zhu, S. Wang, and D. Chen, "Energy-efficient user association for heterogeneous cloud cellular networks,” in Proc. of IEEE Globecom Workshops (GC Wkshps), pp. 273-278, Dec. 2012. Article (CrossRef Link)

[12] Y. Xu, R. Hu, L. Wei, and G. Wu, "QoE-aware mobile association and resource allocation over wireless heterogeneous networks," in Proc. of IEEE Global Commun. Conf. (GLOBECOM), pp. 4695-4701, Dec. 2014. Article (CrossRef Link)

[13] H. Pervaiz, L. Musavian, and Q. Ni, "Joint user association and energy efficient resource allocation with minimum-rate constraints in two-tier hetnets," in Proc. of IEEE 24th Int. Symp. Pers. Indoor Mobile Radio Commun. (PIMRC), pp. 1634-1639, Sep. 2013. Article (CrossRef Link)

[14] L. P. Qian, Y. J. Zhang, Y. Wu, and J. Chen, “Joint base station association and power control via benders’ decomposition,” IEEE Trans.Wireless Commun., vol. 12, no. 4, pp. 1651-1665, Apr. 2013. Article (CrossRef Link)

[15] A. M. Geoffrion, “Generalized benders decomposition,” J. Optim.Theory Appl., vol. 10, no. 4, pp. 1-24, 1972. Article (CrossRef Link)

[16] Y. Chen, S. Zhang, S. Xu, and G. Li, "Fundamental trade-offs on green wireless networks," IEEE Commun. Mag., vol. 49, no. 6, pp. 30-37, Jun. 2011. Article (CrossRefLink)

[17] G. Auer et al., "How much energy is needed to run a wireless network?” IEEE Wireless Commun., vol. 18, no. 5, pp. 40-49, Oct. 2011. Article (CrossRef Link)

[18] D. Feng, C. Jiang, G. Lim, J. Cimini, L.J., G. Feng, and G. Li, “A survey of energy-efficient wireless communications,” IEEE Commun. Surveys Tuts., vol. 15, no. 1, pp. 167-178, First Quart. 2013. Article (CrossRef Link)

[19] J. Rao and A. Fapojuwo, "A survey of energy efficient resource management techniques for multicell cellular networks,” IEEE Commun. Surveys Tuts., vol. 16, no. 1, pp. 154-180, 2014. Article (CrossRef Link)

[20] L. Su, C. Yang, Z. Xu, and A. Molisch, "Energy-efficient downlink transmission with base station closing in small cell networks," in Proc. of IEEE Int. Conf. Acoust. Speech Signal Process. (ICASSP), May 2013, pp. 4784-4788. Article (CrossRef Link)

[21] Y. S. Soh, T. Quek, M. Kountouris, and H. Shin, "Energy efficient heterogeneous cellular 
networks,” IEEE J. Sel. Areas Commun., vol. 31, no. 5, pp. 840-850, May 2013. Article (CrossRef Link)

[22] S. Zhou, J. Gong, Z. Yang, Z. Niu, and P. Yang, "Green mobile access network with dynamic base station energy saving," ACM MobiCom, Beijing, China, Sep.2009. Article (CrossRefLink)

[23] Xiangnan Weng, Dongxu Cao, and Zhisheng Niu, "Energy-Efficient Cellular Network Planning under Insufficient Cell Zooming," in Proc. of IEEE 73rd Vehicular Technology Conference Spring, May 2011. Article (CrossRef Link)

[24] Abdulkafi A A, Kiong T S, Koh J, et al., "Energy efficiency of LTE macro base station," in Proc. of 2012 IEEE International Symposium on Telecommunication Technologies (ISTT), pp. 259- 264, 2012. Article (CrossRef Link)

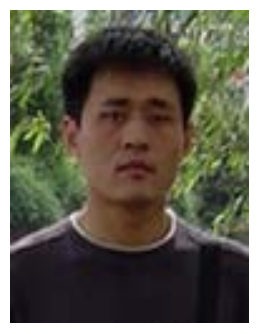

Guolin Sun received his B.S., M.S. and Ph.D. degrees all in Comm. and Info. System from the University of Electronic Sci.\&Tech. of China (UESTC), Chengdu, China, in 2000, 2003 and 2005 respectively. After Ph.D. graduation in 2005, Dr. Guolin has got eight years industrial work experiences on wireless research and development for LTE, Wi-Fi, Internet of Things (ZIGBEE and RFID, etc.), Cognitive radio, Location and navigation. Before he join the School of Computer Science and Engineering, University of Electronic Sci.\&Tech. of China, as an Associate Professor on Aug. 2012, he worked in Huawei Technologies Sweden. Dr. Guolin Sun has filed over 30 patents, and published over 30 scientific conference and journal papers, acts as TPC member of conferences. Currently, he serves as a vice-chair of the 5G oriented cognitive radio SIG of the IEEE (Technical Committee on Cognitive Networks (TCCN) of the IEEE Communication Society. His general research interest is 5G/2020 oriented wireless network, including software defined networks, network function virtualization, wireless networks.

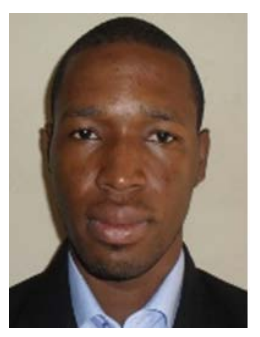

Prince Clement Addo received his Bachelor in Information Technology from University Education, (College of Technology) Kumasi-Ghana, West Africa, in 2014. He is currently Studying MSc. Computer Science and Technology from the University of Electronic Science and Technology of China (UESTC). From 2005 to 2010, he worked as an Instructor and research assistance in Ghana Education Service and CCBTR UEW-K respectively. He is also a member of the Mobile Cloud-Net Research Team - UESTC. His interest include Mobile/Cloud Computing, Internet of Things, 5G and SDN.

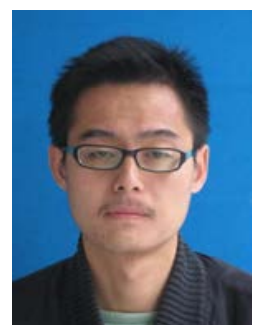

Guohui Wang received Bachelor of Computer Science from Hubei University, China in 2012. He is currently studying MSc in computer science at University of Electronic Science and Technology of China, due to finish in 2016. From 2013 to 2014, he worked as a Software Developer for Open Institute. His interest includes internet of things, SDN, and $5 \mathrm{G}$ architecture.

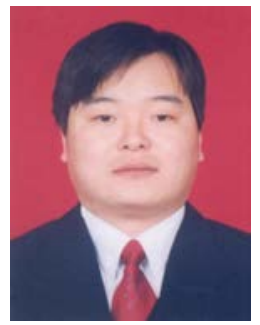

Guisong Liu received his B.S. degree in Mechanics from the Xi'an Jiao Tong University, Xi'an, China, in 1995, M.S. degree in Automatics and Ph.D. degree in Computer Science both from the University of Electronic Science and Technology of China (UESTC), Chengdu, China, in 2000 and 2007 respectively. Now, he is an associated professor in the School of Computer Science and Engineering, UESTC. His research interests include cloud computing, big data, and computational intelligence. 